\title{
Survey on Cardiotocography Feature Extraction Algorithms for Foetal Welfare Assessment
}

\author{
M. Haritopoulos ${ }^{1}$, A. Illanes ${ }^{2}$ and A.K. Nandi ${ }^{3}$ \\ ${ }^{1}$ Univ. Orléans, ENSI de Bourges, PRISME Laboratory, EA 4229, F28000, Chartres, France \\ ${ }^{2}$ Facultad de Ciencias de la Ingeniería, Universidad Austral de Chile, Valdivia, Chile \\ ${ }^{3}$ Electronic and Computer Engineering, Brunel University, Uxbridge, UB8 3PH, UK
}

\begin{abstract}
Since its inception forty years ago as a way to control birth process, the cardiotocograph (CTG) has emerged over time and became the undisputed leader worldwide of noninvasive intrapartum foetal monitoring systems. The CTG signals conveying a lot of information, it is very difficult to interpret them and act accordingly even for specialists; hence, researchers have started looking for characteristics which could be correlated with a particular pathological state of the foetus. Thereby, many features appeared in the literature, ranging from the most common ones to artificially generated features, and computed using a wide variety of signal processing-based analysis tools: time scale, spectral or non-linear analysis, to name but a few. This survey paper, presents in a hierarchical order the most common processing steps of a CTG signal and focuses primarily on the feature extraction methods for foetal heart rate (FHR) analysis reported in the literature during the last decade. Also, some feature classification methods are reported before a brief discussion which concludes this work.
\end{abstract}

Keywords - cardiotocography, feature extraction, classification, foetal heart rate, foetal welfare assessment

\section{INTRODUCTION}

In current clinical practice the assessment of foetal wellbeing during labor and delivery is commonly performed through a non-invasive technique for intrapartum foetal surveillance known as cardiotocograph (CTG), which provides continuous information on foetal heart rate (FHR) in relation with maternal uterine contractions (UC) [1].

The CTG is a simple and non-invasive tool which can provide to clinicians accurate indicators on foetal condition. The ultimate aim of foetal monitoring is to enable clinicians to identify hypoxic foetuses presenting a risk of deterioration to an acidemia in order to prevent potential foetal adverse outcome $[2,3]$ and without excessive obstetric intervention for decreasing maternal and foetal risk.

However, human factors is a critical issue affecting CTG interpretation and clinical management since the analysis of the CTG involves interpretation of the complex relationship between the FHR and the UC signals. A good interpretation of CTG depends on the knowledge, skills and experience of healthcare practitioner to interpret FHR and UC signals in order to recognize the different features and patterns of these signals. Is for this reason that the interpretation of CTG has been shown to suffer from wide intra- and inter- observer disagreement, leading to a poor interpretation reproducibility $[4,5,6,1]$. It has been demonstrated in several works $[7,8,9,3]$ that the observers disagreement involves mainly the identification of variability and decelerations, and the overall tracing classification.

Nowadays, many different solutions have been proposed for improving CTG interpretation and foetal welfare assessment; among the most important are guidelines, which systematically describe the main CTG patterns and the manner they relate with the foetal state. Several medical guidelines for the CTG interpretation have been proposed $[4,9]$. The FIGO guidelines [10] were the first and the only worldwide consensus for CTG interpretation and since then, many other mainly local guidelines produced by national organizations appeared in the literature $[11,12,13]$. However they still lack consensus in many key aspects. The lack of an objective definition of some of the described FHR features and the disparity of definitions between different guidelines are often pointed out in the literature. This leads to good consensus in extreme foetal cases (normal and severe foetal asphyxia) but there is no clear recommendation for cases in between these two extremes which represent at least $50 \%$ of all the intrapartum foetuses $[13,14]$. Moreover guidelines are considered too complex making them prone to rapid memory decay [5].

To overcome these problems other solutions has been proposed, such as CTG-based monitoring by expert systems (ES) and training simulators. Computer-based analysis is today under development and evaluation. Several expert systems has been proposed in the last 20 years $[15,16,17,18]$, and five systems are currently commercialized [19]. As the large majority among these ES use a computerized knowledge database, they just emit visual alerts related to changes in the FHR. In addition, in the recent literature there is little or no evidence that ES have an effect on the incidence of foetal 
acidemia $[20,19]$, caesarean delivery or perinatal mortality, compared with normal CTG analysis. This can be explained by the fact that ES emulate what clinicians do with CTG, increasing the objectivity in the measure, but assuming that the patterns currently used fulfill the set of observations required for correct interpretation.

Simulators can be used to assist clinical staff in the interpretation of CTGs, since they provide a risk-free and controllable environment for training. Several commercial (foetal Sim, iSimulate, NOELLETM, SIMoneTM, and SimMom) and scientific simulators [21, 22, 23, 24] have also been proposed. However, mainstream education is very resourceintensive, and thus, sustained only for limited time periods, after which, the additional knowledge deteriorates [3].

All of the above lead us to consider whether CTG analysis as performed nowadays is optimal in terms of used CTG patterns and guidelines and whether the human interpretation problem is due to insufficient information for analysis and/or is it just a human observation problem. These reasons raise questions on the following points: are the standard morphological time-based CTG extracted patterns sufficient for assessing foetal well-being in terms of foetal acidemia? Does CTG provide all the necessary information for foetal assessment? What is clear is that we do not know what all the patterns of the CTG signal look like a priori. And that is why in recent years many works have been proposed for extracting hidden patterns from the FHR signal using advanced methods for signal processing as spectral or nonlinear analysis, time varying analysis, modeling etc. Most of these methods inherit the methodology already applied to adult HRV signal analysis and thereafter they are adapted to FHR signal analysis, the main idea being to propose new patterns undetectable by the naked eye which can be correlated with the foetal hypoxia problem. It is considered that sets of these hidden patterns added to the standard ones commonly used in the literature can increase CTG specificity.

The overview of the first two stages of a classical CTGs signal processing procedure in section II, will be followed by a survey of feature extraction methods for FHR analysis in CTG recordings in section III, the idea being to make an up to date report of the principal techniques used for the extraction of hidden features proposed the last ten years in the literature. Before concluding this work, a brief state-of-the-art on the essential step of classification according to the previously introduced features will also be given in section III.C.

\section{GENERAL OVERVIEW OF A FHR SIGNAL PROCESSING ALGORITHM}

Most often, a signal processing algorithm for foetal acidemia assessment in CTG recordings involves three main steps: preprocessing, stationary segment extraction, feature extraction and classification. The first two steps will be described here after, while section III will focus on an in depth presentation of the various existing types of features but also on some dedicated techniques for features classification purposes.

\section{A. CTG signal preprocessing}

Clinical CTG recordings usually involve several types of artifacts mainly due to mother's and foetus' movements or displacements of the transducer. The loss of sensor's contact can temporarily interrupt one or both the uterine contraction and the foetal heart rate signals. These artifacts result in sudden changes of short duration in the signal or even in discontinuities thereby causing a complete loss of the signal. The main objective of this step is to detect and to correct outliers in order to analyse reliable continuous signal segments. Generally, the principle for doing this is similar, first of all the signal or adjacent values differences are saturated at values (low FHR and high FHR) that are physiologically inconsistent and then short segment are interpolated and long segment are set to zero. The only main differences between the various algorithms are the threshold values used for the detection and the type of the employed interpolating method [25, 26, 27, 28].

\section{B. Stationary segments extraction}

Due to the FHR signal quality and to discontinuities it is always proposed in the literature to extract stationary or stable segments (called also epochs) from the whole FHR signal. Several epochs' lengths have been proposed depending on the type of the analysis (i.e. time invariant, time variant, short term or long term dynamic analysis $[29,30,31,25,26,32$, 27]). The main principle is that spectral estimation depends on data length. However, the state of the foetus changes with time. Thus, there is a trade-off between selecting an epoch length that is long enough for spectral estimation but also short enough to avoid nonstationarities [26]. In [25, 26] 20 minutes epochs with 10 minutes overlap between successive epochs were used. In [27], segments of 20 minutes are chosen as close as possible to delivery, because during the last minutes major changes in foetal condition can occur. In [32] epochs of 256 heart beats by the use of three sections of 128 beats, each offset by 64 points are taken. In [30, 31], each 
one of the epochs of the time variant analysis is of $32 \mathrm{sec}-$ onds length, and in [29], epochs of 3 minutes are selected for time variant analysis.

\section{CTG FEATURE EXTRACTION AND CLASSIFICATION ALGORITHMS}

Several algorithms for CTG feature extraction have been proposed in the literature, from simple time domain statistical features to more complex time variant and nonlinear features. Clearly, most algorithms are in the field of spectral analysis inspired by research performed in adult HRV. In this section an overview of the existing methods is presented.

\section{A. Time invariant spectral based features}

According to several studies different frequency contributions can be identified in FHR [29, 32]: a DC component (i.e. the average of the FHR), a very low frequency (VLF) band $(0-0.03 \mathrm{~Hz})$ related to very slow control mechanisms and presenting non linear characteristics, a low frequency (LF) band $(0.03-0.15 \mathrm{~Hz})$, mainly associated physiologically with neural sympathetic foetal activity, a high frequency (HF) component $(0.5-1.0 \mathrm{~Hz})$ related to foetal breathing $(\mathrm{HF})$, and also a movement frequency $(\mathrm{MF})(0.15-0.5 \mathrm{~Hz})$ correlated with foetal movements and maternal breathing. Their power spectral densities (PSDs) have been used in previous research work for discriminating foetal pathologies [29].

The frequency-based features are usually computed by using FFT-based techniques over the energy of the different spectral components described above [33, 34, 35, 36, 37, 32]. As in adults HRV, it is assumed that differences in spectral bands can be correlated with foetal acidosis (through the umbilical cord pH) [38]. Spectral analysis allows estimating the effect of the interaction between sympathetic and parasympathetic systems and the alterations of the heart rate variation. However, the low signal-to-noise ratio (SNR) and missing data characterizing CTG recordings make the spectral estimation a difficult task through FFT-based techniques. It is for this reason that AR-based parametric modeling techniques have been proposed for spectral analysis [39] and pole-based features analysis [40]: these latter are known to require only a fraction of the samples needed for the FFT method for the same resolution and to allow the extraction of quantitative spectral parameters.

\section{B. Time variant spectral- and scale-based features}

Standard and advanced time invariant frequency analysis do not take into account the time varying dynamics of the FHR signal and the slow progressive dynamical changes occurring over time within this signal and both resulting from the direct in/out relationship between UC and FHR, since frequency components computing does not take into account the time and the dynamical changes due to this in/out relationship.

In this context, some research works proposed to take into account these dynamical changes through different methodologies focusing on different objectives. Time-frequency methods using Short Time Fourier Transform [31], quadratic time-frequency distributions [41, 42] or time varying AR modelling $[29,30]$ have been used mainly for assessing foetal reactivity through the analysis of FHR as a response to a UC and also for classifying hypoxic and non hypoxic foetuses. For the same aim, Continuous [43] and Discrete Wavelets Transform [44, 45] has been also used in order to take into account the transient nature of the UC excitation.

Other approaches have been proposed focusing on the direct dynamic relationship between UP (as an input) and FHR (as an output), through impulse response modelling [25] or adding to this an AR-based modelling of the FHR variability [26].

\section{Various approaches}

Besides the qualitative approaches of FHR patterns, work based on the quantitative analysis of CTG records exists in the literature. Thus, authors in [46] propose quantitative tools for distress detection from foetal heart rate monitoring; Lomb periodogram, multiscale sample entropy and crosscorrelation of FHR and UC signals offer tools to confirm obstetricians' interpretation of FHR monitoring. The multiscale entropy (MSE) has also been employed in [47] in order to discriminate foetuses with severe acidemia from non-acidemic ones based on the computation of the complexity index of the FHR time series but the last 30 minutes. Another technique applied to the FHR variability of three different groups of foetuses classified according to their $\mathrm{pH}$ values together with the type of their FHR (normal, intermediate or abnormal) is Multifractal Analysis [48]. The analysis was based on wavelet Leader and the obtained results showed that all six parameters were highly correlated with the group of acidotic foetuses and this tool should be very helpful to obstetricians for decision making.

Fractals use has also been reported in [49]; by applying detrended fluctuation analysis (DFA) to FHR data recorded at night, the authors computed the fractal properties and the 
magnitude and sign scaling exponents. They showed that the baseline's parameters values match a multifractal behaviour in its fluctuations and that the magnitude coefficient $\alpha_{(m a g)}$ changes significantly around the $30^{\text {th }}$ week of gestation. Also, in [50], a multiscale multifractal analysis (MMA) - which is a generalisation of the multifractal DFA (MF-DFA) approach, is used to assess the state of the autonomic nervous system (ANS) by predicting the gestational age based on magnetocardiographic recordings. In another work [51], authors explore the dependence of the Short Term Variability (STV) (using the nine most known indexes) - which is relevant of the autonomic nervous system functioning, on the FHR mean value, on the heart rate signal storage rate and on the floatingline changes (accelerations/decelerations).

In [52] significant differences have been shown between complexity indexes for time domain and frequency domain parameters computed on foetal growth restriction (FGR) foetuses after their significance validation based on Wilcoxon rank-sum and t-tests. Authors discussed on the link between the obtained results and the fluximetric indices' alteration but the limited number of studied subjects did not allow to establish a relationship between these results and hypoxia. Work in [53] is also related to the foetal growth and particularly to the intrauterine growth restriction (IUGR) concerning foetuses exposed to a risk of hypoxia. MSE analysis by means of approximate entropy (ApEn) and sample entropy (SampEn), Lempel Ziv complexity analysis, PSD estimation and usual spectral indexes, but also, a multiparametric k-means cluster-based analysis have been tested on real CTGs in order to distinguish between healthy from severe and not severe IUGRs, with the multiparametric approach performing better in clinical applications. Finally, two comparison studies, the first one, between SampEn and ApEn techniques for MSE analysis in order to identify distressed foetuses and, the second one, between linear and non linear indexes in the final 20 minutes preceding the delivery to distinguish normal from acidemic foetuses are reported in [54] and [55], respectively.

Support vector machines (SVMs) have been utilised in [56] for identifying FHR signals from CTG recordings likely to develop metabolic acidosis. The authors have tested different SVM architectures (RBF kernels vs polynomial kernels) to classify the time domain, frequency domain and morphological features previously extracted from the experimental data set and compared their performance by means of the geometric mean $(g)$ and the area under the receiver operating characteristic (ROC) curves (AUC). The area under ROC curves is used also in [57] where wavelet analysis and the evolution of the Hurst parameter describe the temporal dynamics in the first and second stages of labour for normal and acidotic foetuses. Another methodology aiming at the early diagnosis of foetal acidemia is proposed in [58]; in this work, new artificially generated features derived from the actual ones using the grammatical evolution approach are provided as input to a multilayer perceptron (MLP) with one hidden layer nonlinear classifier and, finally, the overall method is validated using 10-fold stratified cross validation method.

An original but no less interesting approach of the missing data recovery on CTGs problem is proposed in [59]. In this work, the authors suggest to use the independent component analysis (ICA) signal processing technique and perform the prediction in the ICA subspace (i.e. after estimation of the independent components (ICs)) using an AR-based linear predictor before projecting the data back to the input space in order to recover the original time series; comparison results with classical linear AR models conclude this work. Also, a quite recent non linear technique, the symbolic dynamic analysis (SDA) has been introduced in [60]. In this work, after computation of the RR series, the authors choose an alphabet of only five symbols leading hence to four word classes to which real CTG data have been classified according to the values of a new variability index (VI) in order to find its possible correlation with spontaneous deliveries (SD) and caesarean section (CS). In a similar work [61], the results obtained with the SDA method have been compared to those obtained by using classical parameters - like morphological features, computed with frequency domain analysis (FDA) tools in order to assess the foetal well-being and development.

A comparison work between linear and non linear features selection for classifying normal and acidotic foetuses is presented in [62]. The authors highlight the fact that the interpretation as well as comparison of the obtained results and their relation with existing research works strongly depend on the approach used to process and to classify the FHR signals. A similar work applied to a different real world CTGs data set and using the RELIEF algorithm for feature space dimensionality reduction and the synthetic minority oversampling technique (SMOTE) to overcome the problem of class imbalance (i.e. much fewer samples in the pathological foetuses class than in the normal foetuses one) is finally presented in [28].

\section{DISCUSSION}

Despite recent updating of medical guidelines on how to use and interpret CTGs and the appearance of various CTG features extraction and classification methods including a large number of triers, there is still controversy about the usefulness of tested methods in the context of clinical use. Aside from the intra- and inter-observer disagreement, some 
progress can be made in terms of understanding of the physiological mechanisms inherent to the foetus defence as well as its adaptation strategy to events occurring during labour.

These slowly evolving events together with other factors, such as the gestational age and the medication administered to the mother, can lead to poor reading and interpretation of the symptoms characterizing foetal distress and, thus, this may be decisive on obstetrician's decision-making trial. One of the main reasons for this fact is that many of the current morphological features extraction approaches are time invariant-based only corresponding to the deceleration patterns of the foetal heart rate tracings. But it is very difficult to say under these conditions what the CTG features are in order to prevent the risk of foetal brain damage.

In this work we wanted pointing out in an non-exhaustive way the current state of the research on CTG signal features extraction based on different approaches, but also to provide a brief overview of various techniques of classification which, once associated to clinician's knowledge and experience on the foetal physiology during labour, could lead to the creation of simple and robust tools for use in connection with caring for the well-being of the foetus.

\section{REFERENCES}

1. Nageotte M P. Fetal heart rate monitoring Seminars in Fetal and Neonatal Medicine. 2015;20:144-148.

2. Campos D Ayres, Ugwumadu A, Banfield P, et al. A randomised clinical trial of intrapartum fetal monitoring with computer analysis and alerts versus previously available monitoring $B M C$ pregnancy and childbirth. 2010;10:71.

3. Hruban L, Spilka J, Chudáček V et al. Agreement on intrapartum cardiotocogram recordings between expert obstetricians Journal of evaluation in clinical practice. 2015.

4. Campos D Ayres, Bernardes J. Twenty-five years after the FIGO guidelines for the use of fetal monitoring: Time for a simplified approach? International Journal of Gynecology \& Obstetrics. 2010;110:1-6.

5. Santo S, Campos D Ayres. Human factors affecting the interpretation of fetal heart rate tracings: an update Current Opinion in Obstetrics and Gynecology. 2012;24:84-88.

6. Devoe L D. Future perspectives in intrapartum fetal surveillance Best Practice \& Research Clinical Obstetrics \& Gynaecology. 2015.

7. Campos D Ayres, Bernardes J. Early, variable and late decelerations: can a consensus be reached in their identification? International Journal of Gynecology \& Obstetrics. 1999;65:305-306.

8. Campos D Ayres, Bernardes J, Marsal K, et al. Can the reproducibility of fetal heart rate baseline estimation be improved? European Journal of Obstetrics \& Gynecology and Reproductive Biology. 2004;112:4954

9. Spilka J, Chudáček V, Janku P, et al. Analysis of obstetricians decision making on CTG recordings Journal of biomedical informatics. 2014;51:72-79.

10. Rooth G, Huch A, Huch R. FIGO News: guidelines for the use of fetal monitoring Int J Gynecol Obstet. 1987;25:159-67.

11. Obstetricians American College, Gynecologists , others . ACOG Practice Bulletin No. 106: Intrapartum fetal heart rate monitoring: nomenclature, interpretation, and general management principles. Obstetrics and gynecology. 2009;114:192.
12. Macones G A, Hankins G DV, Spong C Y, Hauth J, Moore T et al. The 2008 National Institute of Child Health and Human Development workshop report on electronic fetal monitoring: update on definitions, interpretation, and research guidelines Journal of Obstetric, Gynecologic, \& Neonatal Nursing. 2008;37:510-515.

13. Parer J T, Ikeda T. A framework for standardized management of intrapartum fetal heart rate patterns American journal of obstetrics and gynecology. 2007;197:26-e1.

14. Ugwumadu A. Are we (mis) guided by current guidelines on intrapartum fetal heart rate monitoring? Case for a more physiological approach to interpretation BJOG: An International Journal of Obstetrics \& Gynaecology. 2014;121:1063-1070.

15. Pardey J, Mouldenb M, Redman C WG. A computer system for the numerical analysis of nonstress tests American journal of obstetrics and gynecology. 2002;186:1095-1103.

16. Steer P J. Has electronic fetal heart rate monitoring made a difference? in Seminars in Fetal and Neonatal Medicine;13:2-7Elsevier 2008.

17. Graatsma EM, Jacod BC, Egmond LAJ Van, Mulder EJH, Visser GHA et al. Fetal electrocardiography: feasibility of long-term fetal heart rate recordings BJOG: An International Journal of Obstetrics \& Gynaecology. 2009;116:334-338.

18. Parer J T, Hamilton E F. Comparison of 5 experts and computer analysis in rule-based fetal heart rate interpretation American journal of obstetrics and gynecology. 2010;203:451-e1.

19. Nunes I, Campos D Ayres. Computer analysis of foetal monitoring signals Best Practice \& Research Clinical Obstetrics \& Gynaecology. 2015.

20. Lutomski J E, Meaney S, Greene R A, Ryan A C, Devane D. Expert systems for fetal assessment in labour The Cochrane Library. 2013

21. Jagt M B, Oei S G, Bovendeerd P HM. A mathematical model for simulation of early decelerations in the cardiotocogram during labor Medical Engineering \& Physics. 2012;34:579-589.

22. Hout-van M B, Oei S G, Bovendeerd P HM et al. Simulation of reflex late decelerations in labor with a mathematical model Early human development. 2013;89:7-19.

23. Hout-van M B, Jongen G JLM, Bovendeerd P HM, Oei S G et al. Insight into variable fetal heart rate decelerations from a mathematical model Early Human Development. 2013;89:361-369.

24. Illanes A, Haritopoulos M, Robles F, Guerra F. A qualitative dynamical model for cardiotocography simulation in Proceedings of the 42nd Annual Conference Computing in Cardiology;42 2015.

25. Warrick P A, Hamilton E F, Precup D, Kearney R E et al. Identification of the dynamic relationship between intrapartum uterine pressure and fetal heart rate for normal and hypoxic fetuses Biomedical Engineering, IEEE Transactions on. 2009;56:1587-1597.

26. Warrick P A, Hamilton E F, Precup D, Kearney R E et al. Classification of normal and hypoxic fetuses from systems modeling of intrapartum cardiotocography Biomedical Engineering, IEEE Transactions on. 2010;57:771-779.

27. Spilka J, Chudáček V, Kouckỳ M, et al. Using nonlinear features for fetal heart rate classification Biomedical Signal Processing and Control. 2012;7:350-357.

28. Spilka J, Georgoulas G, Karvelis P, et al. Automatic evaluation of FHR recordings from CTU-UHB CTG database in Information Technology in Bio-and Medical Informatics;8060 of 0302-9743:47-61Springer Berlin Heidelberg 2013.

29. Signorini M G, Magenes G, Cerutti S, Arduini D. Linear and nonlinear parameters for the analysisof fetal heart rate signal from cardiotocographic recordings Biomedical Engineering, IEEE Transactions on. 2003;50:365-374

30. Romano M, Bifulco P, Cesarelli M, Sansone M, Bracale M et al. Foetal heart rate power spectrum response to uterine contraction Medical and Biological Engineering and Computing. 2006;44:188-201.

31. Romano M, Bracale M, Cesarelli M, et al. Antepartum cardiotocography: A study of fetal reactivity in frequency domain Computers in biology and medicine. 2006;36:619-633. 
32. Kwon J Y, Park I Y, Shin J C, Song J, Tafreshi R, Lim J et al. Specific change in spectral power of fetal heart rate variability related to fetal acidemia during labor: comparison between preterm and term fetuses Early human development. 2012;88:203-207.

33. Siira S M, Ojala T H, Vahlberg T J, et al. Marked fetal acidosis and specific changes in power spectrum analysis of fetal heart rate variability recorded during the last hour of labour BJOG: An International Journal of Obstetrics \& Gynaecology. 2005;112:418-423.

34. Laar J O Van, Porath M M, Peters C H L, Oei S G. Spectral analysis of fetal heart rate variability for fetal surveillance: review of the literature Acta obstetricia et gynecologica Scandinavica. 2008;87:300-306.

35. Laar J O Van, Peters C H L, Vullings R, Houterman S, Oei S G et al. Power spectrum analysis of fetal heart rate variability at near term and post term gestation during active sleep and quiet sleep Early human development. 2009;85:795-798.

36. Laar J O Van, Peters C H L, Vullings R, Houterman S, Bergmans J W, Oei $\mathrm{S} \mathrm{G}$ et al. Fetal autonomic response to severe acidaemia during labour BJOG: An International Journal of Obstetrics \& Gynaecology. 2010;117:429-437.

37. Laar J O Van, Peters C H L, Houterman S, Wijn P F F, Kwee A, Oei S G et al. Normalized spectral power of fetal heart rate variability is associated with fetal scalp blood $\mathrm{pH}$ Early human development. 2011;87:259-263.

38. Siira S M, Ojala T H, Vahlberg T J, Rosén K G, Ekholm E M et al. Do spectral bands of fetal heart rate variability associate with concomitant fetal scalp pH? Early human development. 2013;89:739-742.

39. Warrick P A, Hamilton E F. Fetal heart-rate variability response to uterine contractions during labour and delivery in Computing in Cardiology (CinC), 2012:417-420IEEE 2012.

40. Illanes A, Haritopoulos M. Fetal Heart Rate Feature Extraction from Cardiotocographic Recordings through Autoregressive Model's Power Spectral- and Pole-based Analysis in Proceedings of the 37th Annual International Conference of the IEEE Engineering in Medicine and Biology Society:5842-5845 2015.

41. Dong S, Azemi G, Boashash B. Improved characterization of HRV signals based on instantaneous frequency features estimated from quadratic time-frequency distributions with data-adapted kernels Biomedical Signal Processing and Control. 2014;10:153-165.

42. Dong S, Boashash B, Azemi G, Lingwood B E, Colditz P B et al. Automated detection of perinatal hypoxia using time-frequency-based heart rate variability features Medical \& biological engineering \& computing. 2014;52:183-191.

43. Spyridou K K, Hadjileontiadis L J. Analysis of fetal heart rate in healthy and pathological pregnancies using wavelet-based features in Engineering in Medicine and Biology Society, 2007. EMBS 2007. 29th Annual International Conference of the IEEE:1908-1911IEEE 2007.

44. Salamalekis E, Siristatidis C, Vasios G, et al. Fetal pulse oximetry and wavelet analysis of the fetal heart rate in the evaluation of abnormal cardiotocography tracings Journal of Obstetrics and Gynaecology Research. 2006;32:135-139.

45. Cattani C, Doubrovina O, Rogosin S, Voskresensky S L, Zelianko E et al. On the creation of a new diagnostic model for fetal well-being on the base of wavelet analysis of cardiotocograms Journal of medical systems. 2006;30:489-494.

46. Cao H, Lake D E, Ferguson J E, Chisholm C A, Griffin M P, Moorman J R et al. Toward Quantitative Fetal Heart Rate Monitoring IEEE Transactions on Biomedical Engineering. 2006;53:111-118.

47. Costa M D, Schnettler W T, Amorin-Costa C, et al. Complexity-loss in fetal heart rate dynamic during labor as a potential biomarker of acidemia Early Human Development. 2014;90:67-71.

48. Doret M, Helgason H, Abry P, Goncales P, Gharib C, Gaucherand P et al. Multifractal Analysis of Fetal Heart Rate Variability in Fetuses with and witout Severe Acidosis during Labor American Journal of Perinatology. 2011;28:259-266.

49. Echeverríaa J C, Álvarez-Ramíreza , Pẽna M A, Rodrígueza E, Gaitánb M J, González-Camarenab R et al. Fractal and nonlinear changes in the long-term baseline fluctuations of fetal heart rate Medical Engineering \& Physics. 2012;34:466-471.

50. Gieratowski J, Hoyer Dirk, Schneider U, Żebrowski J J. Assessment of Fetal Development using Multiscale Multifractal Analysis of Heart Rate Variability in Proceedings of the 40th Annual Conference Computing in Cardiology;40:93-96 2013.

51. Cesarelli M, Romano M, Bifulco P. Comparison of short term variability indexes in cardiotocographic foetal monitoring Computers in Biology and Medicine. 2009;39:106-118.

52. Ferrario M, Magenes G, Campanile M, Carbone I F, Lieto A Di, Signorini M G et al. Multiparameter Analysis of Heart Rate Variability Signal for the Investigation of High Risk Fetuses in 31st Annual International Conference of the IEEE EMBS(Minneapolis, Minnesota, USA):4662-4665 2009.

53. Ferrario M, Signorini M G, Magenes G. Complexity analysis of the fetal heart rate variability: early identification of severe intrauterine growth-restricted fetuses Medical \& Biological Engineering \& Computing. 2009;47:911-919.

54. Ferrario M, Signorini M G, Magenes G, Cerutti S. Comparison of Entropy-Based Regularity Estimators: Application to the Fetal Heart Rate Signal for the Identification of Fetal Distress IEEE Transactions on Biomedical Engineering. 2006;53:119-125.

55. Gonalves H, Rocha A P, Campos D Ayres, Bernardes J. Linear and nonlinear fetal heart rate analysis of normal and acidemic fetuses in the minutes preceding delivery Medical \& Biological Engineering \& Computing. 2006.

56. Georgoulas G, Stylios C D, Groumpos P P. Predicting the Risk of Metabolic Acidosis for Newborns Based on Fetal Heart Rate Signal Classification Using Support Vector Machines IEEE Transactions on Biomedical Engineering. 2006;53:875-884.

57. Spilka J, Abry P, Goncalves P, Doret M et al. Impacts of First and Second Labour Stages on Hurst Parameter based Intrapartum Fetal Heart Rate Analysis in Computing in Cardiology (CinC):777-780 2014.

58. Georgoulas G, Gavrilis D, Tsoulos I G, Stylios C, Bernardes João, Groumpos P P et al. Novel approach for fetal heart rate classification introducing grammatical evolution Biomedical Signal Processing and Control. 2007;2:69-79.

59. Koutras A, Georgoulas G.. Robust Cardiotocogram Prediction using Independent Component Analysis in 5th European Symposium on Biomedical Engineering (ESBME)(Patras, Greece) 2006.

60. Romano M, Bifulco P, Improta G, et al. Symbolic Dynamics in cardiotocographic monitoring in The 4th IEEE International Conference on E-Health and Bioengineering - EHB 2013(Grigore T. Popa University of Medicine and Pharmacy, Iaşi, Romania) 2013.

61. Romano M, Clemente F, D'Addio G, Ponsiglione A M, Importa G, Cesarelli $\mathrm{M}$ et al . Symbolic dynamic and frequency analysis in foetal monitoring in Medical Measurements and Applications (MeMeA), 2014 IEEE International Symposium on:1-5 2014.

62. Spilka J, Chudáček V, Koucký M, et al. Using nonlinear features for fetal heart rate classification Biomedical Signal Processing and Control. 2012;7:350-357.

\section{CONFLICT OF INTEREST}

The authors declare that they have no conflict of interest.

Corresponding author:

Michel Haritopoulos

PRISME Laboratory

Université d'Orléans

21, rue de Loigny la Bataille

28000 Chartres, France

michel.haritopoulos@univ-orleans.fr 